\title{
Diagnostic Imaging of the Diffuse Hepatic Epithelioid Hemangioendothelioma's Type: A Case Report
}

Fabrizio Albarello, Federica Di Stefano, Giovanni Vennarecci, Giuseppe Ettorre, Elisa Busi Rizzi and Paolo Campioni

Universita degli Studi di Ferrara, Cona, Ferrara, Italy

*Corresponding author: Campioni P, Universita degli Studi di Ferrara, Cona, Ferrara, Italy, Tel: +39 3394508689, E-mail: paolo.campioni@unife.it

Rec Date: Jul 20, 2016, Acc Date: Aug 09, 2016, Pub Date: Aug 14, 2016

Copyright: ( 2016 Albarello F, et al. This is an open-access article distributed under the terms of the Creative Commons Attribution License, which permits unrestricted use, distribution, and reproduction in any medium, provided the original author and source are credited.

\begin{abstract}
We present the case of 35 years old female patient who came to our Institute with an unspecified abdominal pain, hepatosplenomegaly and a previous alleged diagnosis of Budd-Chiari syndrome. Magnetic resonance imaging (MRI) was crucial to identify a diffuse and infiltrating mass involving the liver in a misleading diagnosis. A discussion about the imaging findings and differential diagnosis of a rare case of diffuse epithelioid hemangioendothelioma (EHE) is provided as follows. MRI was the most important instrument to guide the diagnostic and therapeutic path, helping to define the typical signal intensity of EHE, mainly with a hepatobiliary contrast agent. The differentiating between other primary tumors, however, remains a complicated issue. Our purpose was to highlight the radiologic and MR characteristics of this rare tumor in order to evaluate the diffuse EHE in the diagnostic process.
\end{abstract}

Keywords: Diffuse hepatic epithelioid hemangioendothelioma; Magnetic resonance; Computed tomography; Ultrasound; Liver

\section{Introduction}

Hepatic epithelioid hemangioendothelioma (HEH) is a rare malignancy of mesenchymal origin with a low to an intermediate grade and an unknown etiology [1]. Definitive diagnosis of $\mathrm{HEH}$ requires histopathologic confirmation and the imaging findings associated with these tumors is often misleading as reported by several authors $[1-3,5,6]$. Since its imaging characteristics resembles more frequent disease (e.g. metastasis), the HEH should be always considered in the differential of diffuse infiltrating masses of the liver. Our purpose was to describe the CT and MR appearances of this tumor with a particular focus on the application of the diffusion weighted imaging and the hepatobiliary contrast agent.

\section{Case Presentation}

On December 2014, a 35 years old woman was received at our radiology department, with non-specific symptoms including right upper quadrant pain, hepatomegaly and weight loss. She also reported a previous Ultrasonography (US) where the liver was found with diffuse heterogeneous pattern, hyper/isoechoic pseudo nodular areas, mainly on the peripheral region. The study of the hepatic vein flow and webs was reported to be unreliable due to meteoric artifacts. The patient was addressed to our department with an alleged Budd-Chiari syndrome.

In order to exclude sovrahepatic obstruction, the hepatic venous pressure gradient (HVPG) was measured but no pressure variations nor inferior caval vein (ICV) stenosis were observed (wedged pressure: $8 \mathrm{mmHg}$; free pressure: $5 \mathrm{mmHg}$; ICV: $3.5 \mathrm{mmHg}$; intrahepatic IVC: $2.5 \mathrm{mmHg}$; right atrium: $2 \mathrm{mmHg}$ ).

The physical examination and the esophagus-gastro-duodenoscopy did not reveal direct or indirect signs of portal hypertension. The liver function test showed higher level of alkaline phosphatase in the serum.
The patient underwent abdominal US examination with an iU22 system (Philips, Netherlands) equipped with a $2 \mathrm{MHz}$ to $5 \mathrm{MHz}$ convex array probe. US confirmed the coarse inhomogeneity of the liver with no veins obstructions (Figure 1).

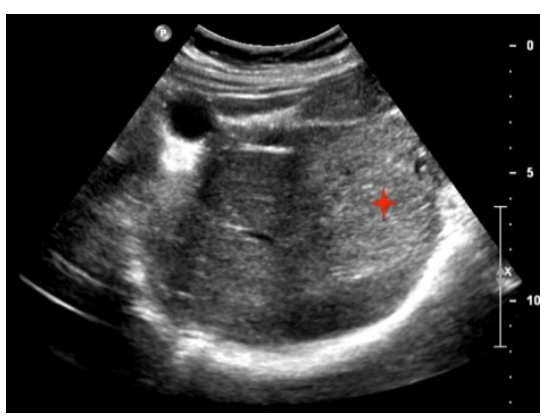

Figure 1: US of the liver showing a diffuse heterogeneous pattern, hyper/isoechoic pseudo nodular areas, mainly on the peripheral region (red star).

Consecutively, a MRI of the abdomen was performed at $1.5 \mathrm{~T}$ (Signa, GE Healthcare, Milwaukee, Wisconsin), using a 8 channel body phased array surface coil. MRI analysis was performed with T1weighted in-phase and out-of-phase, T2-weighted fast spin echo (FSE), Diffusion Weighted Imaging (DWI, b 50-1000), sequences, on the axial and coronal planes, with and without fat saturation, completed with sequences after intravenous administration of $0,1 \mathrm{mg} / \mathrm{kg}$ of paramagnetic contrast media (Gd-BOPTA - Multihance -Bracco, Italy).

Dynamic contrast enhanced fat suppressed T1 weighted images were obtained at 25, 65 and 120 seconds after contrast agent infusion. Additionally, 1 hour delayed hepatobiliary images were acquired. The presence of confluent and diffuse mass, extending to the capsular margin within an enlarged liver, associated with capsular retraction, was highlighted. 
The lesions showed a heterogeneous hypointense signal in the T1 weighted images and slight hyperintense to the liver parenchyma in the long TR sequences. The in-phase and out-of-phase sequences excluded the presence of a fatty liver infiltration and fat within the lesion.

After intravenous administration of the contrast agent, a patchy and inhomogeneous enhancement was observed. The lesions showed a centripetal contrast filling during the dynamic late phase, with hypointense signal in the hepatobiliary phase, after 1 hour. The DWI displayed a restricted signal on the peripheral region of the liver (Figure 2a-2h).

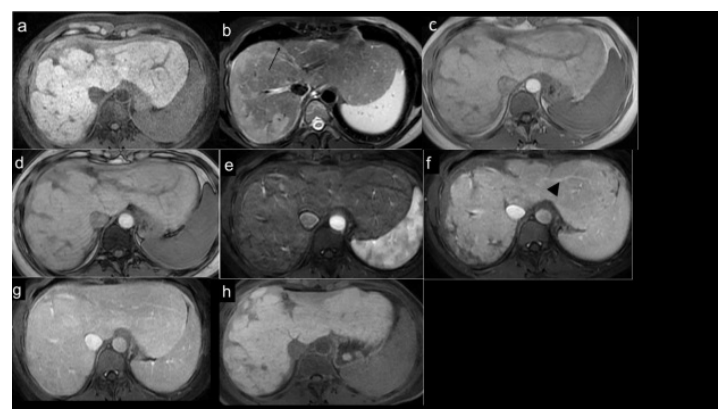

Figure 2: Axial T1 (a) and T2 TSE (b) weighted images illustrate an enlarged liver with coalescing and diffuse areas, extending to the capsular margin, associated with capsular retraction (arrow). No intracellular fat was seen in the GRE in and out of phase sequences $(\mathrm{c}, \mathrm{d})$. Axial Gd-BOPTA-enhanced T1 weighted image in arterial (e), portal (f) and $180 \mathrm{~s}$ delayed (g) phase shows a slow and in homogenous enhancement without washout in the equilibrium phase of the peripheral region. The lesions present low signal at the hepatobiliary phase (h). Note the peripheral displacement of the main portal vessels (arrowhead). The tumor presents a high restricted signal on the liver edge at b $50 \mathrm{~mm} / \mathrm{sec}^{2}$ on DWI.

Moreover, a peripheral displacement of the main portal vessels was noted. Nor obstructions of the porto-mesenteric system neither of the main hepatic veins were seen.

Thus, MRI showed a dystrophic liver with irregular shape associated to peripheral atrophic parenchyma, mainly in the III, IV segment and in the anterior sections. A consistent hypertrophy of the I and II segment could simulate a Budd Chiari disease as well as a spontaneous hyperintense T2 weighted signal and an alternation of hypertrophic and atrophic areas due to the vascular impairment. However, it was caused by the peripheral infiltration of the tumor which arrives under the capsule with a progressive enhancement and displaces the hepatic veins. Peripheral vein was free such us the right and left branches of the portal vein. On the other hand, the portal vessels within the most atrophic segments were almost vanished.

The hypothesis of an epithelioid hemangioendothelioma (HEH) with a diffuse pattern was addressed but histopathologic confirmation was still needed. Histological specimen was obtained by ultrasound guided percutaneous needle biopsy. HEH was diagnosed based on light microscopic examination and both on hematoxylin-eosin staining and immunohistochemically staining results.

Microscopically, the tumor consisted of proliferated fibrous tissue intermingled with epithelioid cells. Subsequently, a four-phase contrast-enhanced total body CT (Bright Speed, GE Healthcare,
Milwaukee, Wisconsin; 32-slice, thickness: $3 \mathrm{~mm}$; delay: 35-75-180 sec; $100 \mathrm{ml}$ of non-ionic organ iodate contrast agent at $4.5 \mathrm{ml} / \mathrm{s}$ Iopamigita $370 \mathrm{~g} / \mathrm{L}$ - Agfa Healthcare) was performed to stage the tumor and for the surgical planning.

Multiple hepatic lesions, forming confluent masses involving both hepatic lobes, were confirmed. Most of those lesions were distributed peripherally on the right lobe with compensatory hypertrophy of the left lobe and the caudate lobe. The blank phase showed diffuse hypoattenuated areas as compared with the surrounding liver parenchyma.

Contrast-enhanced CT's findings included marginal enhancement during the arterial phase, becoming more intense during the portal and equilibrium phase (Figure 3a-3f).

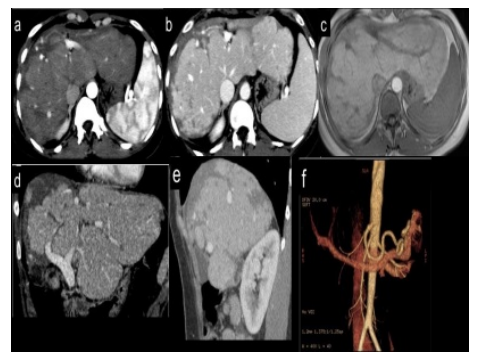

Figure 3: Axial post contrast CT slices showing the infiltration of the dysplastic tissue into the liver $(\mathrm{a}-\mathrm{c})$. Note that the lesion confirms the slow enhancement which is higher in the delayed phase (c). Coronal and sagittal MPR in the portal phase confirmed the extension of the tumor $(\mathrm{d}, \mathrm{e})$. Volume rendering reconstruction, displaying the tripod and the splenic-portal axis, was useful for the surgical planning (f).

No other suspected lesions were detected. Since patients with this kind of HEH have a variable evolution, especially in such infiltrating pattern, this patient was addressed for liver transplantation and she is currently on the transplant list.

\section{Discussion}

$\mathrm{HEH}$ is a rare primary malignancy of mesenchymal origin, with an estimated annual incidence of fewer than 1 per 1.000 .000 [1-4]. Approximately, $60 \%$ of the affected patients are women (female to male ratio of 3:2), with a peak of incidence occurring between 30 and 40 years of age $[5,6]$.

No specific causes have been identified and, according to the World Health Organization (WHO) classification, HEH is a tumor of variable malignant potential [7]. Histopathological, $\mathrm{HEH}$ consists of epithelioid and dendritic cells with central fibrotic stroma.

Neoplastic endothelial cells invade and, eventually, obliterate sinusoids, terminal hepatic veins and portal veins. Its predilection for intravascular growth is responsible for the infarction of the tumor resulting in fibrosis, progressive sclerosis and eventual calcifications [8]. Three forms of $\mathrm{HEH}$ have been described: single nodular, multifocal nodular and diffuse [2-4].

The latter one is considered to be, according to some authors, the end-stage of a multifocal nodular pattern which is going to infiltrate the parenchyma $[6,9]$. 
Detection of HEH is usually incidental, since most of the patients are asymptomatic or present with non-specific symptoms such as upper right abdominal pain and weight loss. Moreover, liver function tests are frequent abnormal but not specific [1,9-11].

Definitive diagnosis of $\mathrm{HEH}$ requires histopathologic confirmation but radiologists should be aware of the imaging findings associated with these tumors in order to facilitate an accurate diagnostic work-up.

Early stage recognition of this rare tumor can facilitate treatments such as resection or transplantation [4]. While surgical resection has been validated as a first choice of treatment in resectable cases with no extrahepatic involvement, most cases are unresectable at diagnosis, due to the multicentricity of the disease. In this case, the most common treatment is liver transplantation [12].

Regarding the difficulty of diagnosing this disease, we point out the different imaging features in order not to miss this kind of lesion; the possible differential diagnosis includes:

- Peripheral Cholangiocarcinoma: it may present as a heterogeneous mass with capsular retraction as the confluent nodes of the $\mathrm{HEH}$ "Satellite" lesions may be seen but usually, not as many as with HEH and it is often associated with intrahepatic bile duct dilatation;

- Focal Confluent Fibrosis: despite the presence of a dense fibrous stroma could resemble the diffuse $\mathrm{HEH}$, it is commonly seen in advanced cirrhosis;

- Hemangioma (in a cirrhotic liver): the typical blood pool could be missed in a cirrhotic liver. Generally, after hepatospecific contrast agent, it presents diffuse and central enhancement.

- Treated Malignancy (e.g. hepatocellular carcinoma or metastases): the history of the patient is mandatory in this case. Capsular retraction with a heterogeneous enhancement pattern may be seen. Treated metastatic nodules may show cystic or necrotic changes with thick septa and wall enhancement;

- Budd-Chiari syndrome: it can complicate an $\mathrm{HEH}$, due to tumor invasion of the hepatic veins in the advanced stage of disease;

- Veno-occlusive disease: imaging findings frequently reveal larger liver veins that may have decreased caliber but which are nevertheless still evident $[1,4,11,13-16]$.

Focusing back to our case, we found a diffuse and massive tumor, extending from the left lobe to the right anterior segment, infiltrating the sinusoid and determining a compensatory hypertrophy of the normal liver, characteristically of the caudatus lobe (Figures 2 and 3).

The typical three concentric layers of alterated signal intensity, as described by Bruegel at al. was not seen in our case [17]. Neither the different target patterns described by Paoloantonio et al. were observed [18]. Even though we found a peripheral distribution with nodules coalescence and capsular retraction, as previously described in literature, the diffuse lesions showed a non-characteristic and misdiagnosing enhancement pattern in the dynamic MR phases $[1,10,11,19,20]$.

The hepatospecific phase played a crucial role in the differential diagnosis, mainly in differentiating the peripheral cholangiocarcinoma: the latter, usually, shows peripheral hypointensity but central iso-or hypointensity on biliar-specific phase, unlike the $\mathrm{HEH}$, which in our case, presented homogeneous dismissing of GdBOPTA after one hour [21,22].
Several studies reported that during the hepatospecific phase, the $\mathrm{HEH}$ may presents a contrast enhanced core due to an entrapment of contrast agent within the fibrotic tissue and a surrounding "halo" sign of hypointensity due to the lack of functional hepatocytes in the peripheral edge of the tumor $[18,22]$. Nevertheless, in the present case, the whole tumor had a homogeneous low signal in the biliary phase. It could depend to the different biological behaviors of the tumor during its evolution.

Regarding the Budd-Chiari disease, Stark et al. already reported how the dynamic MRI of the liver may display an increased arterial perfusion of the affected regions and a prolonged liver transit time of contrast agent [23]. In our patient, despite the presence of portosystemic shunts, Budd-Chiari syndrome was excluded: generally, the patchy areas of enhancement surround the main portal branches and not only the liver edge; moreover, uniform or peripheral delayed retention is seen bright on biliar-specific phase and caudate lobe enhances at the early phase whereas peripheral liver enhances at the later phase (flip-flop sign) [24].

In a recent paper, Gan et al. [25] reported two cases of diffuse HEH with the typical "target -like" sign on non-contrast enhanced MRI, and a heterogeneous behavior with a "strip-like" enhancement in the dynamic phases using Gd-DTPA. Nevertheless, the use of a hepatobiliary contrast media could provide more information's for the differential diagnosis.

The multiparametric study of the liver should always include DWI and ADC maps: the high signal intensity related to the restricted diffusion can help the radiologist to easily locate the tumor [17] mainly in the diffuse subtype of $\mathrm{HEH}$.

According to the literature, the tumor extension is better defined in the unenhanced CT scan, because sometimes the enhanced phase could underestimate the real tumor size when is lacking the avascular area around the tumor (causing the typical target sign) [21].

However, the MRI was the most important instrument to guide the diagnostic and therapeutic path: the dynamic phases were necessary to study the vascular behavior of the masses but the best diagnostic tool was the hepatospecific phase, necessary to confirm the absence of functioning hepatocyte within the lesion. DWI could be useful for increasing the confidence in recognizing the restricted areas as malignant.

\section{References}

1. Makhlouf HR, Ishak KG, Goodman ZD (1999) Epithelioid hemangioendothelioma of the liver: a clinicopathologic study of 137 cases. Cancer 85: 562-582.

2. Amin S, Chung H, Jha R (2011) Hepatic epithelioid hemangioendothelioma: MR imaging findings. Abdom Imaging 36: 407-414.

3. Azzam RI, Alshak NS, Pham HP (2012) AIRP best cases in radiologicpathologic correlation: Hepatic epithelioid hemangioendothelioma. Radiographics 32:789-794.

4. Mehrabi A, Kashfi A, Fonouni H, Schemmer P, Schmied BM, et al. (2006) Primary malignant hepatic epithelioid hemangioendothelioma: a comprHEHnsive review of the literature with emphasis on the surgical therapy. Cancer 107: 2108-2121.

5. Earnest Ft, Johnson CD (2006) Case 96: Hepatic epithelioid hemangioendothelioma. Radiology 240: 295-298.

6. Furui S, Itai Y, Ohtomo K, Yamauchi T, Takenaka E, et al. (1989) Hepatic epithelioid hemangioendothelioma: report of five cases. Radiology 171: 63-68. 
Citation: Albarello F, Stefano FD, Vennarecci G, Ettorre G, Rizzi EB, et al. (2016) Diagnostic Imaging of the Diffuse Hepatic Epithelioid Hemangioendothelioma's Type: A Case Report. J Clin Case Rep 6: 849. doi:10.4172/2165-7920.1000849

Page 4 of 4

7. Flejou JF (2011) [WHO Classification of digestive tumors: the fourth edition]. Ann Pathol 31: 27-31.

8. Requena L, Kutzner H (2013) Hemangioendothelioma. Semin Diagn Pathol 30: 29-44.

9. Lyburn ID, Torreggiani WC, Harris AC, Zwirewich CV, Buckley AR, et al. (2003) Hepatic epithelioid hemangioendothelioma: sonographic, CT, and MR imaging appearances. AJR Am J Roentgenol 180: 1359-1364.

10. Miller WJ, Dodd GD, Federle MP, Baron RL (1992) Epithelioid hemangioendothelioma of the liver: imaging findings with pathologic correlation. AJR Am J Roentgenol 159: 53-57.

11. Weiss SW, Enzinger FM (1982) Epithelioid hemangioendothelioma: a vascular tumor often mistaken for a carcinoma. Cancer 50: 970-981.

12. Lerut JP, Orlando G, Adam R, Schiavo M, Klempnauer J, et al. (2007) The place of liver transplantation in the treatment of hepatic epithelioid hemangioendothelioma: report of the European liver transplant registry. Ann Surg 246: 949-957.

13. Buetow PC, Buck JL, Ros PR, Goodman ZD (1994) Malignant vascular tumors of the liver: radiologic-pathologic correlation. Radiographics 14: 153-166.

14. Lakshmi SV, Prabhavathy D, Jayakumar S, Janaki C, Tharini GK (2012) Epithelioid hemangioendothelioma: a rare vascular tumor. Indian J Dermatol 57: 53-54.

15. Lamba R, Fananapazir G, Corwin MT, Khatri VP (2014) Diagnostic imaging of hepatic lesions in adults. Surg Oncol Clin N Am 23: 789-820.

16. Lin J, Ji Y (2010) CT and MRI diagnosis of hepatic epithelioid hemangioendothelioma. Hepatobiliary Pancreat Dis Int 9: 154-158.
17. Bruegel M, Muenzel D, Waldt S, Specht K, Rummeny EJ (2011) Hepatic epithelioid hemangioendothelioma: findings at CT and MRI including preliminary observations at diffusion-weighted echo-planar imaging. Abdom Imaging 36: 415-424.

18. Paolantonio P, Laghi A, Vanzulli A, Grazioli L, Morana G, et al. (2014) MRI of hepatic epithelioid hemangioendothelioma (HEH). J Magn Reson Imaging 40: 552-558.

19. Radin DR, Craig JR, Colletti PM, Ralls PW, Halls JM (1988) Hepatic epithelioid hemangioendothelioma. Radiology 169: 145-148.

20. Uchimura K, Nakamuta M, Osoegawa M, Takeaki S, Nishi H, et al. (2001) Hepatic epithelioid hemangioendothelioma. J Clin Gastroenterol 32: 431-434.

21. Schneidez (2006) Epithelioid Hemangioendothelioma. MRI of the LiverImaging techniques Contrast Enhancement Differential Diagnosis. Springer 211-214.

22. Kim EH, Rha SE, Lee YJ, Yoo Ie R, Jung ES, (2015) CT and MR imaging findings of hepatic epithelioid hemangioendotheliomas: emphasis on single nodular type. Abdom Imaging. 40: 500-509.

23. Stark DD, Hahn PF, Trey C, Clouse ME, Ferrucci JT (1986) MRI of the Budd-Chiari syndrome. AJR Am J Roentgenol 146: 1141-1148.

24. Buckley O, J OB, Snow A, Stunell H, Lyburn I, et al. (2007) Imaging of Budd-Chiari syndrome. Eur Radiol 17: 2071-2078.

25. Gan L, Chang R, Jin H, Yang L (2016) Typical CT and MRI signs of hepatic epithelioid hemangioendothelioma. Oncology letters 11: 1699-1706. 